TRANSACTIONS OF THE

AMERICAN MATHEMATICAL SOCIETY

Volume 288, Number 2, April 1985

\title{
NO $L_{1}$-CONTRACTIVE METRICS FOR SYSTEMS OF CONSERVATION LAWS ${ }^{1}$
}

BY

\author{
BLAKE TEMPLE
}

Abstract. Let

(*)

$$
u_{t}+F(u)_{x}=0
$$

be any $2 \times 2$ system of conservation laws satisfying certain generic assumptions on $F$ in a neighborhood $\mathscr{N}$ of $u$-space. We prove that for every nondegenerate metric $D$ on $u$-space there exists states $u_{1}$ and $u_{2}$ in $\mathscr{N}$ such that $\int_{-\infty}^{\infty} D\left(u(x, t), u_{1}\right) d x$ is a strictly increasing function of $t$ in a neighborhood of $t=0$, where $u$ is the admissible solution of $(*)$ with initial data

$$
u(x, 0)= \begin{cases}u_{1}, & x \leqslant 0, \\ u_{2}, & 0<x<1, \\ u_{1}, & x \geqslant 1 .\end{cases}
$$

This contrasts with the case of a scalar equation in which $\int_{-\infty}^{\infty} D(u(x, t), v(x, t)) d x$ is a decreasing function of $t$ for all admissible solution pairs $u$ and $v$ when $D$ is taken to be the absolute value norm.

\section{Introduction. Let}

$$
u_{t}+F(u)_{x}=0, \quad u \equiv u(x, t),
$$

be any $2 \times 2$ system of conservation laws which is strictly hyperbolic and genuinely nonlinear (cf. [8]) in both characteristic fields in some open neighborhood $\mathscr{N}_{0}$ of a state $u_{0} \in \mathbf{R}^{2}$. Assume also that the shock and rarefaction curves do not coincide in at least one characteristic family, say the first (cf. [14]). (By [14] this is equivalent to assuming that each integral curve to the first eigenvector of $d F$ is not a straight line in any open subset of $\mathscr{N}_{0}$; i.e., $(r \cdot \nabla) r \neq 0$ in $\mathscr{N}_{0}$ is sufficient, where $r$ is an eigenvector field, $|r|=1$.) We prove that for such systems there does not exist a metric $D$ compatible with state space such that

$$
\int_{-\infty}^{+\infty} D(u(x, t), v(x, t)) d x
$$

is a decreasing function of time for all physical weak solutions $u$ and $v$ that agree off a compact set.

More precisely we prove the stronger statement that there does not exist a metric that is $L^{1}$-contractive relative to a constant state for a simple class of noninteracting solutions. In particular, let $d F$ denote the matrix derivative of $F$ with respect to $u$, let

Received by the editors August 25, 1983.

1980 Mathematics Subject Classification. Primary 35L65, 76N10.

${ }^{1}$ Work done while author was a visiting member at the Courant Institute. 
$\lambda_{1} \equiv \lambda_{1}(u)<\lambda_{2}(u) \equiv \lambda_{2}$ denote the eigenvalues of $d F$, and let

$$
\Lambda=\sup \left\{\left|\lambda_{2}(u)-\lambda_{1}(v)\right|, u, v \in \mathscr{N}_{0}\right\} \text {. }
$$

Let

$$
u_{0}\left(\varepsilon, u_{1}, u_{2} ; x\right)= \begin{cases}u_{1} & \text { for }-\infty \leqslant x \leqslant 0 \\ u_{2} & \text { for } 0<x \leqslant \varepsilon \\ u_{1} & \text { for } \varepsilon<x \leqslant+\infty\end{cases}
$$

The Riemann problems posed in (1)and $\left(1^{\prime}\right)$ can be solved and are noninteracting for times $t \leqslant \Lambda^{-1} \varepsilon$ (cf. [8]). Let $u\left(\varepsilon, u_{1}, u_{2} ; x, t\right)$ denote this solution, $0 \leqslant t \leqslant \Lambda \varepsilon$. We prove the following theorem.

THEOREM 1. Let $u$ be any weak solution of the form

$$
u(x, t)=u\left(\varepsilon, u_{1}, u_{2} ; x, t\right) .
$$

Then there does not exist a metric $D$ compatible with $u$-space such that

$$
\int_{-\infty}^{\infty} D\left(u(x, t), u_{1}\right) d x
$$

is a decreasing function of time for all $u_{1}, u_{2} \in \mathcal{N}_{0}, 0 \leqslant \varepsilon \leqslant \Lambda, 0 \leqslant t \leqslant \Lambda^{-1} \varepsilon$.

By a metric compatible with $u$-space we mean any symmetric function

$$
D: \mathscr{N}_{0} \times \mathscr{N}_{0} \rightarrow \mathbf{R}
$$

such that, for all $u, v$ and $w$ in $\mathscr{N}_{0}$,

$$
\text { (triangle inequality) } \quad D(u, v)+D(v, w) \geqslant D(u, w)
$$

and

$$
\text { (compatibility) } \quad C_{0}^{-1}|u-v| \leqslant D(u, v) \leqslant C_{0}|u-v|
$$

for some uniform constant $C_{0}>0$.

Because of the scale invariance of (1), the following corollary is a direct consequence of Theorem 1 (see the Appendix).

COROllary 1. There does not exist a constant $\omega$ and a metric $D$ compatible with $u$-space such that either of the following Gronwall-type inequalities holds for all weak solutions

$$
u(x, t) \equiv u\left(\varepsilon, u_{1}, u_{2} ; x, t\right),
$$

where $u_{1}, u_{2} \in \mathscr{N}_{0}, 0 \leqslant \varepsilon \leqslant \Lambda$ and $0 \leqslant t \leqslant \Lambda^{-1} \varepsilon$ :

$$
\begin{aligned}
& \frac{d}{d t} \int_{-\infty}^{\infty} D\left(u(x, t), u_{1}\right) d x \leqslant \omega \int_{-\infty}^{\infty} D\left(u(x, t), u_{1}\right) d x \\
& \int_{-\infty}^{\infty} D\left(u(x, t), u_{1}\right) d x \leqslant e^{\omega t} \int_{-\infty}^{\infty} D\left(u(x, 0), u_{1}\right) d x
\end{aligned}
$$

Moreover, because the class of functions given in (7) contains only noninteracting solutions, we can also conclude from Theorem 1 that there does not exist a metric such that (2) will decrease during each iteration of the random choice method of Glimm (cf. [3]). 
This result for systems contrasts with the case of a scalar conservation law for which the integral (2) is decreasing when $D$ is taken to be the usual absolute value norm (cf. [6, 15]). In particular, Theorem 1 implies that such a result for systems will fail when $D$ is taken to be the absolute value norm in any variables which are obtained by a regular transformation of the conserved variables.

We remark that the class of systems for which the shock and rarefaction curves do not coincide in one family includes the equations of elasticity and gas dynamics, but excludes certain equations that arise in chemical engineering (cf. $[1,4,5,7,10,13$, 14]). Restricting to this class ensures that the system does not uncouple, in which case an $L_{1}$-contractive metric does exist by results on scalar equations.

We prove Theorem 1 by contradiction, i.e., we obtain a set of inequalities by assuming that (3) decreases for certain solutions of form (7). These inequalities are then shown to be inconsistent with our other assumptions.

2. Proof of the theorem. To prove Theorem 1 , assume that $D$ is an $L_{1}$-contractive metric for a $2 \times 2$ system (1) that satisfies our other assumptions. The four lemmas to follow are used to obtain a contradiction, and thus a proof of Theorem 1.

We adopt the following notation. Let $r_{i} \equiv r_{i}(u), i=1,2$, denote the eigenvectors of the matrix $d F(u)$ for corresponding eigenvalues $\lambda_{i}(u), i=1,2$. Let $R_{i}(u)$ denote the integral curve of $r_{i}(u)$ in $\mathscr{N}_{0}$, and for $v \in R_{i}(u)$, let $R_{i}(u, v)$ denote the set of points on $R_{i}(u)$ that lie strictly between $u$ and $v$. Let $S_{1}(u)$ denote the 1-shock curve in $\mathscr{N}_{0}$ associated with the left-hand state $u$. Such curves exist locally in $\mathscr{N}_{0}$, and for $v \in S_{1}(u), \lambda_{1}(v) \leqslant \lambda_{1}(u)$ (cf. [8]). Our assumption that the shock and rarefaction curves do not coincide in the first family states that $S_{1}(u) \not \subset R_{1}(u)$ for any $u \in \mathscr{N}_{0}$. By taking $\mathscr{N}_{0}$ sufficiently small we can, without loss of generality, assume that (cf. [14]) $\mathscr{N}_{0} \cap S_{1}(u) \cap R_{1}(u)=\{u\}$.

We now let $u, \bar{u}, v, \bar{v}$ denote any states in $\mathscr{N}_{0}$ related in the following way (see Figure 1):

$$
\bar{v} \in S_{1}(u), \quad\{v\}=R_{1}(u) \cap R_{2}(\bar{v}), \quad\{\bar{u}\}=R_{2}(u) \cap R_{1}(\bar{v}),
$$

and such that all states on the various shock and integral curves of $r_{i}$ that connect these points pairwise also lie in $\mathscr{N}_{0}$. In this case we say that $u, \bar{u}, v, \bar{v}$ have the configuration of Figure 1. For example, by our assumptions, in any neighborhood of $u \in \mathscr{N}_{0}$ there exist states $\bar{u}, v$ and $\bar{v}$ such that $u, \bar{u}, v, \bar{v}$ have the configuration of Figure 1.

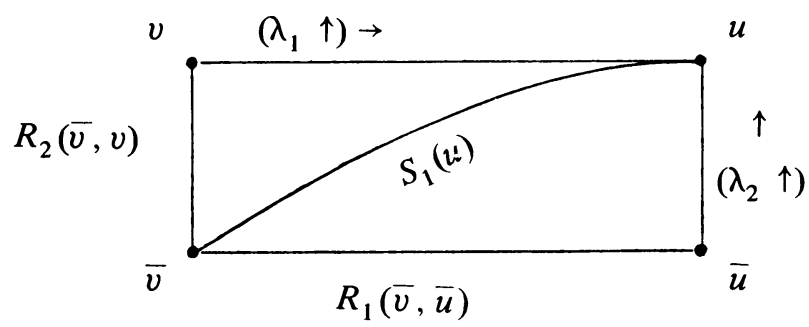

FigURE 1 


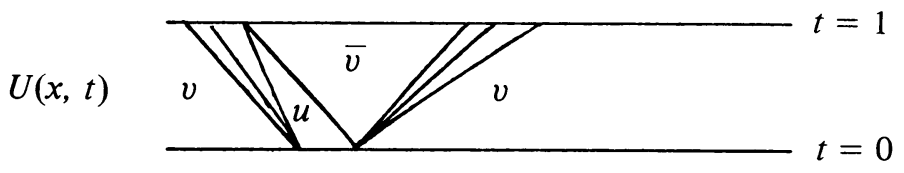

FIGURE 2

If $u, \bar{u}, v, \bar{v}$ have the configuration of Figure 1 , then by strict hyperbolicity and genuine nonlinearity, $\lambda_{1}$ increases monotonically from $v$ to $u$ and from $\bar{v}$ to $\bar{u}$ along $R_{1}(v, u)$ and $R_{1}(\bar{v}, \bar{u})$, respectively. Without loss of generality assume further that $\lambda_{2}$ increases monotonically from $\bar{v}$ to $v$ and from $\bar{u}$ to $u$ along $R_{2}(\bar{v}, v)$ and $R_{2}(\bar{u}, u)$, respectively. (If $\lambda_{2}$ decreases on $R_{2}(\bar{v}, v)$ and $R_{2}(\bar{u}, u)$, then the following argument goes through with straightforward modifications. By our assumptions of strict hyperbolicity and genuine nonlinearity, one of these two monotonicity assumptions must occur for all $u, \bar{u}, v$, and $\bar{v}$ in $\mathscr{N}_{0}$ if $\mathscr{N}_{0}$ is sufficiently small.) Let $\mu(\lambda)$ (resp. $\bar{\mu}(\lambda)$ ) denote the parametrization of $R_{1}(v, u)$ (resp. $\bar{\mu}(\bar{v}, \bar{u})$ ) with respect to $\lambda_{1}$, and let $\nu(\lambda)$ (resp. $\bar{\nu}(\lambda)$ ) denote the parametrization of $R_{2}(\bar{v}, v)$ (resp. $R_{2}(\bar{u}, u)$ ) with respect to $\lambda_{2}$. Finally, let $s$ denote the speed of the 1-shock that connects $u$ to $\bar{v}$.

LEMMA 1. The following inequalities hold for any states $u, \bar{u}, v, \bar{v}$ which have the configuration of Figure 1:

$$
\begin{aligned}
& \int_{\lambda_{1}\left(v^{\prime}\right)}^{\lambda_{1}(u)}[D(\mu(\lambda), u)+D(\mu(\lambda), v)] d \lambda-\left(\lambda_{1}(u)-\lambda_{1}(v)\right) D(u, v) \geqslant 0 \\
& \int_{\lambda_{1}(\bar{u})}^{\lambda_{1}(\bar{u})}[D(\bar{\mu}(\lambda), u)+D(\bar{\mu}(\lambda), \bar{v})] d \lambda-\left(\lambda_{1}(\bar{u})-\lambda_{1}(\bar{v})\right) D(u, \bar{v}) \geqslant 0 .
\end{aligned}
$$

Proof. By the triangle inequality,

$$
D(\mu(\lambda), u)+D(\mu(\lambda), v) \geqslant D(u, v)
$$

for all $\mu(\lambda) \in R_{1}(v, u)$. Integrating (11) from $\lambda_{1}(v)$ to $\lambda_{1}(u)$ verifies (10a). Statement $(10 b)$ is derived similarly.

Lemma 2. There exists a neighborhood $\mathscr{N}_{1}$ of $u_{0}$ and a constant $C_{1}>0, \mathscr{N}_{1} \subset \mathscr{N}_{0}$, such that, if $u, \bar{u}, v, \bar{v}$ are states in $\mathscr{N}_{1}$ and have the configuration of Figure 1, then

$$
D(\bar{v}, u)-D(v, u) \leqslant-C_{1}|u-\bar{u}| .
$$

Proof. Consider first the following solution $U$ defined for $0 \leqslant t \leqslant 1$ :

$$
U(x, 0)= \begin{cases}u & \text { for } x \in\left[0, \lambda_{1}(u)-s\right] \\ v & \text { otherwise }\end{cases}
$$

Let

$$
I_{1}(t)=\int_{-\infty}^{\infty} D(u(x, t), v) d x
$$

Then

$$
\begin{aligned}
I_{1}(1)= & \int_{\lambda_{1}\left({ }^{\prime \prime}\right)}^{\lambda_{1}(u)} D(\mu(\lambda), v) d \lambda+\left(\lambda_{2}(\bar{v})-\lambda_{1}(\bar{v})\right) D(\bar{v}, v) \\
& +\int_{\lambda_{2}(\bar{v})}^{\lambda_{2}\left({ }^{\prime \prime}\right)} D(\nu(\lambda), v) d \lambda
\end{aligned}
$$




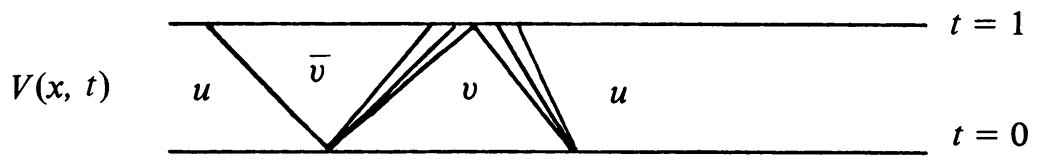

FIGURE 3

and

(16)

$$
I_{1}(0)=\left(\lambda_{1}(u)-s\right) D(u, v) .
$$

But by our assumption, $I_{1}(1)-I_{1}(0) \leqslant 0$, so we have

$$
\int_{\lambda_{1}(v)}^{\lambda_{1}(u)} D(\mu(\lambda), v) d \lambda \leqslant\left(\lambda_{1}(u)-s\right) D(u, v)-\left(\lambda_{2}(\bar{v})-\lambda_{1}(\bar{v})\right) D(\bar{v}, v) .
$$

We now obtain a similar estimate for a complementary solution $V$ defined for $0 \leqslant t \leqslant 1$ :

$$
V(x, 0)= \begin{cases}v & \text { for } x \in\left[0, \lambda_{2}(v)-\lambda_{1}(v)\right] \\ u & \text { otherwise }\end{cases}
$$

Let

$$
I_{2}(t)=\int_{-\infty}^{\infty} D(V(x, t), u) d x
$$

$$
I_{2}(1)=\left(\lambda_{2}(\bar{v})-s\right) D(\bar{v}, u)+\int_{\lambda_{2}(\bar{v})}^{\lambda_{2}(v)} D(\nu(\lambda), u) d \lambda+\int_{\lambda_{1}(v)}^{\lambda_{1}(u)} D(\mu(\lambda), u) d \lambda
$$

and

$$
\begin{aligned}
I_{2}(0) & =\left(\lambda_{2}(v)-\lambda_{1}(v)\right) D(v, u) \\
& =\left[\left(s-\lambda_{1}(v)\right)+\left(\lambda_{2}(\bar{v})-s\right)+\left(\lambda_{2}(v)-\lambda_{2}(\bar{v})\right)\right] D(v, u) .
\end{aligned}
$$

Thus $I_{2}(1)-I_{2}(0) \leqslant 0$ implies

$$
\begin{aligned}
\int_{\lambda_{1}(v)}^{\lambda_{1}(u)} & D(\mu(\lambda), u) d \lambda \\
\leqslant & \left(s-\lambda_{1}(v)\right) D(v, u)+\left(\lambda_{2}(\bar{v})-s\right)[D(v, u)-D(\bar{v}, u)] \\
& +\left\{\left(\lambda_{2}(v)-\lambda_{2}(\bar{v})\right) D(v, u)-\int_{\lambda_{2}(\bar{v})}^{\lambda_{2}(v)} D(\nu(\lambda), u) d \lambda\right\} .
\end{aligned}
$$

Adding (17) to (22) gives

$$
\begin{gathered}
\left\{\int_{\lambda_{1}(v)}^{\lambda_{1}(u)}[D(\mu(\lambda), u)+D(\mu(\lambda), v)] d \lambda-\left(\lambda_{1}(u)-\lambda_{1}(v)\right) D(u, v)\right\} \\
\leqslant\left(s-\lambda_{2}(\bar{v})\right)[D(\bar{v}, u)-D(v, u)]-\left(\lambda_{2}(\bar{v})-\lambda_{1}(\bar{v})\right) D(\bar{v}, v) \\
+\left\{\left(\lambda_{2}(v)-\lambda_{2}(\bar{v})\right) D(v, u)-\int_{\lambda_{2}(\bar{v})}^{\lambda_{2}(v)} D(\nu(\lambda), u) d \lambda\right\}
\end{gathered}
$$

But by (10a) of Lemma 1, the left-hand side of (23) is positive, so we obtain (24)

$$
\begin{aligned}
\left(\lambda_{2}(\bar{v})-s\right)[D(\bar{v}, u)- & D(v, u)] \leqslant-\left(\lambda_{2}(\bar{v})-\lambda_{1}(\bar{v})\right) D(\bar{v}, v) \\
& +\left\{\left(\lambda_{2}(v)-\lambda_{2}(\bar{v})\right) D(v, u)-\int_{\lambda_{2}(\bar{v})}^{\lambda_{2}(v)} D(\nu(\lambda), u) d \lambda\right\} .
\end{aligned}
$$




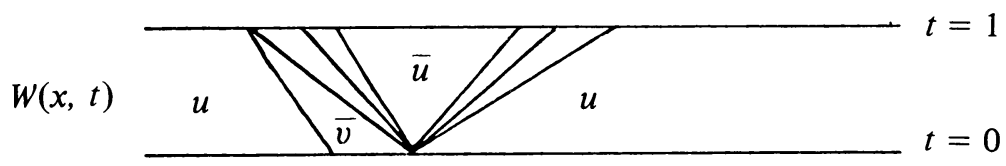

FIGURE 4

Now since $D$ is continuous (a consequence of the compatibility assumption), it is uniformly continuous in a neighborhood of $u_{0}$. Thus for $\tilde{v}=\nu(\lambda), \lambda_{2}(\bar{v}) \leqslant \lambda \leqslant$ $\lambda_{2}(v)$, we can write

$$
D(\tilde{v}, u) \geqslant D(v, u)-\varepsilon(\delta),
$$

where $\varepsilon(\delta) \rightarrow 0+$ as $\delta \rightarrow 0$ when we restrict the states $u, \bar{u}, v, \bar{v}$ to lie in a neighborhood $\mathscr{N}(\delta)$ of $u_{0}$,

$$
\mathscr{N}(\delta) \equiv\left\{W:\left|w-u_{0}\right|<\delta\right\} .
$$

Therefore,

$$
-\int_{\lambda_{2}(\bar{v})}^{\lambda_{2}(v)} D(\nu(\lambda), u) d \lambda \leqslant-\left(\lambda_{2}(v)-\lambda_{2}(\bar{v})\right) D(u, v)+\varepsilon(\delta)\left(\lambda_{2}(v)-\lambda_{2}(\bar{v})\right)
$$

and we can rewrite $(24)$ as

$$
D(\bar{v}, u)-D(v, u) \leqslant-\frac{\lambda_{2}(\bar{v})-\lambda_{1}(\bar{v})}{\lambda_{2}(\bar{v})-s} D(\bar{v}, v)+C_{3} \varepsilon(\delta)|v-\bar{v}|,
$$

where we have used the fact that

$$
0 \leqslant \frac{\lambda_{2}(v)-\lambda_{2}(\bar{v})}{\lambda_{2}(\bar{v})-s} \leqslant C_{3}|v-\bar{v}|,
$$

a consequence of the assumptions of strict hyperbolicity and genuine nonlinearity. Also as consequences of these assumptions, we have

$$
\frac{\lambda_{2}(\bar{v})-\lambda_{1}(\bar{v})}{\lambda_{2}(\bar{v})-s}>C_{4}>0
$$

and

$$
C_{5}^{-1}|\bar{u}-u| \geqslant|\bar{v}-v| \geqslant C_{5}|\bar{u}-u|,
$$

where $C_{i}$ are uniform in $\mathcal{N}_{0}$. Moreover, by the compatibility assumption,

$$
D(\bar{v}, v) \geqslant C_{0}^{-1}|\bar{v}-v|,
$$

so substituting (20)-(31) into (27) yields

$$
D(\bar{v}, u)-D(v, u) \leqslant-C_{4} C_{5} C_{0}^{-1}|\bar{u}-u|+C_{3} C_{5}^{-1} \varepsilon(\delta)|\bar{u}-u| .
$$

Thus there is a $\delta_{1}>0$ and a constant $C_{1}>0$ such that

$$
D(\bar{v}, u)-D(v, u) \leqslant-C_{1}|\bar{u}-u|
$$

whenever $u, v, \bar{u}, \bar{v}$ have the configuration of Figure 1 and also lie in $\mathscr{N}\left(\delta_{1}\right) \equiv \mathscr{N}_{1}$. This completes the proof of Lemma 2. 


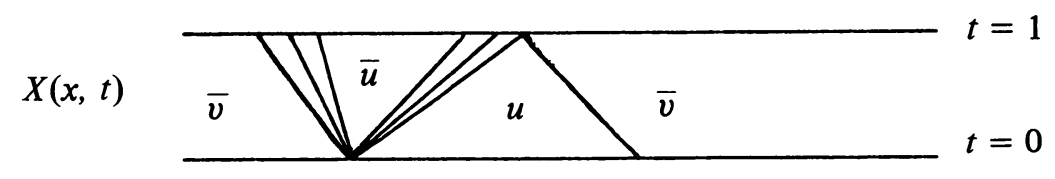

FIGURE 5

LEMMA 3. There exists a neighborhood $\mathscr{N}_{2}$ of $u_{0}$ and a constant $C_{2}>0, \mathscr{N}_{2} \subset \mathscr{N}_{0}$, such that, if $u, \bar{u}, v$ and $\bar{v}$ are states in $\mathscr{N}_{2}$ and have the configuration of Figure 1 , then

$$
D(\bar{u}, \bar{v})-D(u, \bar{v}) \leqslant-C_{2}|\bar{u}-u| .
$$

Proof. As in the proof of Lemma 2, consider first the following solution $W$ defined for $0 \leqslant t \leqslant 1$ :

$$
W(x, 0)= \begin{cases}\bar{v} & \text { for } x \in\left[0, s-\lambda_{1}(\bar{v})\right] \\ u & \text { otherwise }\end{cases}
$$

Let

$$
I_{3}(t)=\int_{-\infty}^{\infty} D(W(x, t), u) d x
$$

Then

$$
\begin{gathered}
I_{3}(1)=\int_{\lambda_{1}(\bar{v})}^{\lambda_{1}(\bar{u})} D(\bar{\mu}(\lambda), u) d \lambda \\
+\left(\lambda_{2}(\bar{u})-\lambda_{1}(\bar{u})\right) D(\bar{u}, u)+\int_{\lambda_{2}(\bar{u})}^{\lambda_{2}(u)} D(\bar{\nu}(\lambda), u) d \lambda \\
I_{3}(0)=\left(s-\lambda_{1}(\bar{v})\right) D(\bar{v}, u) .
\end{gathered}
$$

By our assumption $I_{3}(1)-I_{3}(0) \leqslant 0$, so

$$
\int_{\lambda_{1}(\bar{v})}^{\lambda_{1}(\bar{u})} D(\bar{\mu}(\lambda), u) d \lambda-\left(s-\lambda_{1}(\bar{v})\right) D(\bar{v}, u) \leqslant-\left(\lambda_{2}(\bar{u})-\lambda_{1}(\bar{u})\right) D(\bar{u}, u) .
$$

We now obtain a similar estimate for a complementary solution $X$ defined for $0 \leqslant t \leqslant 1$ :

$$
X(x, 0)= \begin{cases}u & \text { for } x \in\left[0, \lambda_{2}(u)-s\right] \\ \bar{v} & \text { otherwise }\end{cases}
$$

Let

$$
I_{4}(t)=\int_{-\infty}^{\infty} D(X(x, t), \bar{v}) d x
$$

Then

$$
\begin{aligned}
I_{4}(1)= & \int_{\lambda_{1}(\bar{v})}^{\lambda_{1}(\bar{u})} D(\bar{\mu}(\lambda), \bar{v}) d \lambda \\
& +\left(\lambda_{2}(\bar{u})-\lambda_{1}(\bar{u})\right) D(\bar{u}, \bar{v})+\int_{\lambda_{2}(\bar{u})}^{\lambda_{2}(u)} D(\bar{v}(\lambda), \bar{v}) d \lambda, \\
I_{4}(0)= & \left(\lambda_{2}(u)-s\right) D(u, \bar{v}) \\
= & {\left[\left(\lambda_{1}(\bar{u})-s\right)+\left(\lambda_{2}(\bar{u})-\lambda_{1}(\bar{u})\right)+\left(\lambda_{2}(u)-\lambda_{2}(\bar{u})\right)\right] D(u, \bar{v}) . }
\end{aligned}
$$


Thus $I_{4}(1)-I_{4}(0) \leqslant 0$ implies

$$
\begin{aligned}
\int_{\lambda_{1}(\bar{v})}^{\lambda_{1}(\bar{u})} & D(\bar{\mu}(\lambda), \bar{v}) d \lambda-\left(\lambda_{1}(\bar{u})-s\right) D(u, \bar{v}) \\
\leqslant & \left(\lambda_{2}(\bar{u})-\lambda_{1}(\bar{u})\right)[D(u, \bar{v})-D(\bar{u}, \bar{v})] \\
& +\left\{\left(\lambda_{2}(u)-\lambda_{2}(\bar{u})\right) D(u, \bar{v})-\int_{\lambda_{2}(\bar{u})}^{\lambda_{2}(u)} D(\bar{v}(\lambda), \bar{v}) d \lambda\right\} .
\end{aligned}
$$

Adding (39) to (44) and dividing by $\lambda_{2}(\bar{u})-\lambda_{1}(\bar{u})$ gives

$$
\begin{aligned}
& \frac{1}{\lambda_{2}(\bar{u})-\lambda_{1}(\bar{u})}\left\{\int_{\lambda_{1}(\bar{v})}^{\lambda_{1}(\bar{u})} D(\bar{\mu}(\lambda), u) d \lambda+\int_{\lambda_{1}(\bar{v})}^{\lambda_{1}(\bar{u})} D(\bar{\mu}(\lambda), \bar{v}) d \lambda\right. \\
& \leqslant D(u, \bar{v})-D(\bar{u}, \bar{v})-D(u, \bar{u}) \\
& \left.-\left(\lambda_{1}(\bar{u})-\lambda_{1}(\bar{v})\right) D(u, \bar{v})\right\} \\
& +\frac{1}{\lambda_{2}(\bar{u})-\lambda_{1}(\bar{u})}\left\{\left(\lambda_{2}(u)-\lambda_{2}(\bar{u})\right) D(u, \bar{v})-\int_{\lambda_{2}(\bar{u})}^{\lambda_{2}(u)} D(\bar{\nu}(\lambda), \bar{v}) d \lambda\right\} .
\end{aligned}
$$

The left-hand side of (45) is positive by (10b) of Lemma 1 , so we obtain (46)

$$
\begin{aligned}
D(\bar{u}, \bar{v})-D(u, \bar{v}) \leqslant & -D(u, \bar{u})+\frac{1}{\lambda_{2}(\bar{u})-\lambda_{1}(\bar{u})} \\
& \times\left\{\left(\lambda_{2}(u)-\lambda_{2}(\bar{u})\right) D(u, \bar{v})-\int_{\lambda_{2}(\bar{u})}^{\lambda_{2}(u)} D(\bar{\nu}(\lambda), \bar{v}) d \lambda\right\} .
\end{aligned}
$$

But by uniform continuity, $D(\bar{\nu}(\lambda), \bar{v})=D(u, \bar{v})+\varepsilon(\delta)$, so the last term in (46) can be estimated as $O(1) \varepsilon(\delta)|u-\bar{u}|$ as in the proof of Lemma 2. This term is dominated by $-D(u, \bar{u})$ in (46) for $\delta$ sufficiently small, so the proof of Lemma 3 is complete.

Lemma 4. There exists a constant $C>0$ such that, if $u, \bar{u}, v, \bar{v}$ are states in $\mathscr{N} \equiv \bigcap_{i=0}^{2} \mathscr{N}_{i}$ and have the configuration of Figure 1 , then

$$
D(\bar{u}, \bar{v})-D(v, u) \leqslant-C|u-\bar{u}| .
$$

Proof. This follows immediately from Lemmas 2 and 3.

Proof of Theorem 1 . We use Lemma 4 to obtain a contradiction. Let $u_{0}, u_{1}, v_{0}, v_{1}$ in $\mathscr{N}$ have the configuration of Figure 1 in the order $u, \bar{u}, v, \bar{v}$. Assume that $\left|u_{0}-v_{0}\right|$ is sufficiently small so that there is a point $\tilde{u} \in \mathscr{N} \cap R_{2}\left(u_{0}\right)$ on the same side of $u_{0}$ as $u_{1}$, such that

$$
\left|u_{0}-\tilde{u}\right|>\left(C_{0} / C\right)\left|u_{0}-v_{0}\right|
$$

(see (6) for definition of the constants $\left.C, C_{0}\right)$. Let $\{\tilde{v}\}=R_{2}\left(v_{0}\right) \cap R_{1}(\tilde{u})$. By choosing $\left|u_{0}-v_{0}\right|$ sufficiently small, we can assume that the closure of the region bounded by the curves $R_{1}\left(v_{0}, u_{0}\right), R_{1}(\tilde{v}, \tilde{u}), R_{2}\left(v_{0}, \tilde{v}\right), R_{2}\left(u_{0}, \tilde{u}\right)$ lies in $\mathscr{N}$. Now construct the sequence of points $\left\{u_{i}, v_{i}\right\}$ in $\mathscr{N}$ such that

$$
\begin{aligned}
& \left\{v_{i}\right\}=S_{1}\left(u_{i-1}\right) \cap R_{2}\left(v_{i-1}\right), \\
& \left\{u_{i}\right\}=R_{1}\left(v_{i}\right) \cap R_{2}\left(u_{i-1}\right) .
\end{aligned}
$$


Thus for each $i, u_{i-1}, u_{i}, v_{i-1}, v_{i}$ have the configuration of Figure 1. Moreover, by our assumption that the shock and rarefaction curves diverge in the first family, we can assume (by taking $\left|u_{0}-v_{0}\right|$ sufficiently small if necessary) that there is an integer $N>0$ such that $u_{i} \in R_{2}\left(u_{i-1}, \tilde{u}\right)$ for $i<N$, and

$$
\tilde{u} \in R_{2}\left(u_{0}, u_{N}\right) \text {. }
$$

Now we can apply Lemma 4 as follows:

$$
D\left(u_{i}, v_{i}\right)-D\left(u_{i-1}, v_{i-1}\right) \leqslant-C\left|u_{i}-u_{i-1}\right|,
$$

so, summing from 1 to $N$,

$$
\begin{aligned}
\sum_{i=1}^{N} D\left(u_{i}, v_{i}\right)-D\left(u_{i-1}, v_{i-1}\right) & =D\left(u_{N}, v_{N}\right)-D\left(u_{0}, v_{0}\right) \\
& \leqslant-C \sum_{i=1}^{N}\left|u_{i}-u_{i-1}\right| \leqslant-C \cdot \frac{C_{0}}{C}\left|u_{0}-v_{0}\right|
\end{aligned}
$$

or

$$
\begin{aligned}
D\left(u_{N}, v_{N}\right) & \leqslant D\left(u_{0}, v_{0}\right)-C_{0}\left|u_{0}-v_{0}\right| \\
& \leqslant C_{0}\left|u_{0}-v_{0}\right|-C_{0}\left|u_{0}-v_{0}\right| \leqslant 0 .
\end{aligned}
$$

Thus $u_{N}=v_{N}$ by the compatibility assumption. But this is a contradiction, since $u_{N} \in R_{2}\left(u_{0}\right)$ and $v_{N} \in R_{2}\left(v_{0}\right)$, and $R_{2}\left(u_{0}\right) \cap R_{2}\left(v_{0}\right) \cap \mathscr{N}_{0}=\varnothing$ by the assumption of strict hyperbolicity. This completes the proof of Theorem 1.

It is interesting to note that the only use of the triangle inequality is in Lemma 1. Moreover, (10a) follows without the triangle inequality by using the fact that smooth solutions can be run backwards, and thus (2) must be constant in time for smooth solutions, in order for Theorem 1 to fail. Similar methods do not seem to apply to estimate (11b).

Note also that we need not assume that $D$ is symmetric, since if (2) decreases for $\bar{D}$ not symmetric, then (2) decreases for $D(u, v)=\bar{D}(u, v)+\bar{D}(v, u)$, where $D$ is symmetric.

ACKNowledgement. I am indebted to Ronald DiPerna for suggesting this problem to me, and for many helpful discussions and ideas. I also thank Bradly Plohr for reading the first draft.

Appendix. We show that Corollary 1 follows directly from Theorem 1 . Let $D$ be any metric compatible with $u$-space. Assume that $u$ and $v$ are weak solutions of (1) that agree off a compact set in $x$ at $t=0$, such that

$$
\int_{-\infty}^{\infty} D(u(x, T), v(x, T)) d x-\int_{-\infty}^{\infty} D(u(x, 0), v(x, 0)) d x=d>0
$$

for some $T>0$. Let $U_{c}(x, t)=u(c x, c t), V_{c}(x, t)=v(c x, c t)$ and define

$$
I_{c}(t)=\int_{-\infty}^{\infty} D\left(U_{c}(x, t), V_{c}(x, t)\right) d x
$$

Then

$$
I_{c}(t / c)=I_{1}(t) / c \text {. }
$$


Moreover, (A1) reads

(A4)

$$
I_{1}(T)-I_{1}(0)=d>0
$$

and so by (A3)

$$
I_{c}(T / c)-I_{c}(0)=(1 / c)\left[I_{1}(T)-I_{1}(0)\right]=d / c .
$$

Thus

$$
I_{c}(T / c)=I_{c}(0)+d / c=(1 / c)\left[I_{1}(0)+d\right] .
$$

Now let $u$ be a solution of form (7), let $v(x, t) \equiv u_{1}$ and assume there exists $\omega>0$ such that (8) holds for all such $u$. Then since $U_{c}(x, t)$ is also of form (7) with a rescaled value of $\varepsilon$, we must have

$$
I_{c}(T / c) \leqslant e^{\omega T / c} I_{c}(0)
$$

for all $c>1$, and by (A6)

$$
\frac{1}{c}\left[I_{1}(0)+d\right] \leqslant e^{\omega T / c} I_{c}(0)=e^{\omega T / c} \frac{1}{c} I_{1}(0)
$$

or

$$
d \leqslant\left(e^{\omega T / c}-1\right) I_{1}(0) .
$$

But for $c$ sufficiently large, (A9) fails, thus proving the corollary.

\section{REFERENCES}

1. R. Aris and N. Amundson, Mathematical methods in chemical engineering, Vol. 2, Prentice-Hall, Englewood Cliffs, N. J., 1966.

2. R. Courant and K. O. Friedricks, Supersonic flow and shock waves, Wiley, New York, 1948.

3. J. Glimm, Solutions in the large for nonlinear hyperbolic systems of equations, Comm. Pure Appl. Math. 18 (1965), 697-715.

4. E. Isaacson, Global solution of a Riemann problem for a non-strictly hyperbolic system of conservation laws arising in enhanced oil recovery, J. Comp. Phys. (to appear).

5. F. Helfferich and G. Klein, Multicomponent chromatography, Dekker, New York, 1970.

6. B. Keyfitz, Solutions with shocks: An example of an $L_{1}$-contractive semigroup, Comm. Pure Appl. Math. 24 (1971), 125-132.

7. B. Keyfitz and H. Kranzer, A system of non-strictly hyperbolic conservation laws arising in elasticity theory, Arch. Rational Mech. Anal. 72 (1980), 219-241.

8. P. D. Lax, Hyperbolic systems of conservation laws. II, Comm. Pure Appl. Math. 19 (1957), 537-566.

9. Shock waves and entropy, Contributions to Nonlinear Functional Analysis (E. H Zarantonello, ed.), Academic Press, New York, 1971, pp. 603-634.

10. T. P. Liu and C. H. Wang, On a hyperbolic system of conservation laws which is not strictly hyperbolic, MRC Technical Summary Report \#2184, December 29, 1980.

11. D. W. Peaceman, Fundamentals of numerical reservoir simulation, Elsevier North-Holland, New York, 1977.

12. H. Rhee, R. Aris and N. R. Amundson, On the theory of multicomponent chromatography, Philos. Trans. Roy. Soc. London Ser. A 267 (1970), 419.

13. B. Temple, Global solution of the Cauchy problem for a class of $2 \times 2$ non-strictly hyperbolic conservation laws, Adv. in Appl. Math. 3 (1982), 335-375.

14. . Systems of conservation laws with invariant submanifolds, Proc. Amer. Math. Soc. (to appear).

15. M. Walsh, S. Bryant, R. Schechter and L. Lake, Precipitation and dissolution of solids attending flow through porous media, University of Texas Preprint, 1982.

Courant Institute of Mathematical Sciences, New York University, New York, New York 10012

Current address: 2510 Kendall Ave., Madison, Wisconsin 53705 